\title{
Epidemiology and Associated Injuries in Paediatric Femoral Shaft Fractures Treated at a Limited Resource Zonal Referral Hospital in Northern Tanzania
}

\author{
Albert Paul Macha ( $\sim$ albertpaul2013@gmail.com ) \\ Muhimbili Orthopaedic Institute \\ Rogers Joachimu Temu \\ Kilimanjaro Christian Medical University College \\ Frank Olotu \\ Kilimanjaro Christian Medical Centre \\ Neil Sheth \\ University of Pennsylvania \\ Honest Herman Massawe \\ Kilimanjaro Christian Medical University College
}

\section{Research Article}

Keywords: Orthopaedic Injuries, Childhood, Fracture, Femur

Posted Date: April 30th, 2021

DOI: https://doi.org/10.21203/rs.3.rs-385890/v1

License: (c) (1) This work is licensed under a Creative Commons Attribution 4.0 International License.

Read Full License 


\section{Abstract}

Background: Femoral shaft fractures contribute up to $40 \%$ of paediatric orthopaedic admissions with the World Health Organisation data showing youth are particularly vulnerable and road traffic injuries are the leading cause of death for children and young adults. The mechanism of injury varies with age and geographical location of the patient and involves a variety of mechanisms from falls to road traffic injuries. Understanding the incidence, mechanism and pattern of these injuries allows planning for preventive measures and treatment to meet modern day patient demands, generation of appropriate and timely protocols with minimum social and economic burden to the patient and family.

Objectives and Methods: A hospital based cross sectional study was conducted using the orthopaedic department patient registry among children aged under 18 years admitted from 2014 - 2018. Our research question was to determine the epidemiology of femoral shaft fractures and coexisting associated injuries among admitted paediatric orthopaedic patients. Patient files were reviewed from the medical records department and a data collecting sheet was used to record demographics and injury data. Odds ratios with $95 \%$ confidence intervals for associated injuries in paediatric femoral shaft fractures were estimated using multivariable logistic regression model.

Results: We found the prevalence of femoral shaft fracture among paediatric orthopaedic admissions was $18 \%$ with the majority 111 (68.5\%) being males. The most common injury mechanism was a fall (57.4\%) followed by road traffic injuries with $35.8 \%$ of which $48.3 \%$ resulted from pedestrian vs motorcycle accidents. Traumatic brain injury (TBI) was the most common associated injuries accounting for $69 \%$ of these injuries with the majority $79 \%$ occurring in patients aged 6 years and older. When comparing 6-12 years and 13-18 years age groups to those younger $\leq 2$ years, they had 8 and 11 times higher odds for associated injuries (OR 8.25, 95\% Cl, 1.04 - 65.31) $\mathrm{p}=0.046$ and $(\mathrm{OR} 10.54,95 \% \mathrm{Cl}, 1.26$ 88.31) $p=0.031$ respectively with road traffic related injuries having 17 times higher odds of associated injuries when compared to fall (OR 16.73, 95\% Cl, 6.28 - 44.57) $p<0.001 .112(69.1 \%)$ were treated by nonoperative method out of this $90(55.6 \%)$ by traction with delayed Spica application. The overall mean duration of hospital stay was $18.5 \pm 11$ days, ranging from $3-68$ days.

Conclusion: Pedestrian vs motorcycle injuries was the leading specific cause of paediatric femoral shaft fractures with TBI being the common associated injury. Non-operative management was the most utilized treatment plan and contributed to ten times higher odds for a longer duration of hospital stay. Initiatives to insure children safety on roads should be strengthened in order to reduce/eliminate this burden. Application and practice of current evidence based clinical guidelines and recommendations is paramount for timely and appropriate treatment of these injuries.

\section{Introduction}

The World Health Organization (WHO) endorse injury as the leading cause of morbidity and mortality in children after their first year of life. ${ }^{1}$ Femoral shaft fractures account for up to $40 \%$ of paediatric 
orthopaedic admissions in low and middle income countries. ${ }^{2}$ The mechanism of injury for these fractures vary with patient age; falls from standing height or playground equipment are more in younger children while high energy injuries are more commonly seen in adolescents and older children. ${ }^{2,3}$ Recent studies report the significant rise in road traffic injuries (RTI) as the major cause of these fractures. ${ }^{3-5}$

Associated bodily injuries are common in children that sustain a femoral shaft fracture. High energy injury mechanisms increase the risk of associated injuries; and the presence of an associated injury portends a two-fold increase in the hospital length of stay (LOS) irrespective of the treatment modality

employed. ${ }^{6-8}$ Understanding the mechanism of injury and the resulting pattern of paediatric femoral fractures is essential to determine the risk of associated injuries among children. Currently, limited information is available regarding the magnitude and trends of this clinical scenario in sub-Saharan Africa.

We conducted a retrospective audit of over five years to determine the mechanism of injury of paediatric femoral shaft fractures and associated injuries at a zonal referral-teaching hospital in northern Tanzania. Our research question was to determine the prevalence of femoral shaft fractures and coexisting associated injuries among admitted paediatric orthopaedic patients. Defining the magnitude of this problem will be a platform for initiating preventive measures, planning interventions and allocating resources to deliver the best evidence-based patient care..$^{9-11}$

\section{Material And Methods}

The institutional review Ethical and Research Committee approved the study with certificate number 2333. We performed a retrospective, cross-sectional study of all paediatric orthopaedic patients with femoral shaft fractures from January 2014 to December 2018 admitted to the Orthopaedic ward at a zonal referral teaching hospital in northern Tanzania. Located in Moshi district-Tanzania, it is the third largest hospital in the country with 700 official inpatient beds often stretched to accommodate about 100-150 more patients on canvas beds as it servers the country's northern corridor with an estimated catchment population of 15 million people per the national census data. ${ }^{12}$

The Orthopaedic ward admission registry was reviewed for all patients admitted during the study review period. All patients aged 18 years and younger were identified by their diagnosis and further classified by name and file identification number if diagnosed with a femur fracture. Files for patients with a femur fracture were retrieved from the medical records department and were sub-categorized based on fracture location and type of fracture (pathological versus traumatic). All patients with incomplete data were excluded from the study.

A standardized data extraction form was designed to record all demographic and pertinent injury data including age and gender of the patient, admission date, injury mechanisms, site of the fracture, type of associated injury, fracture type, fracture location, treatment type and duration of hospital stay. Collected data were processed and analysed using SPSS (Statistical Package for Social Sciences) software version 
22 (IBM Corp., Armonk, NY, USA). Frequencies, percentages, mean and median were calculated and summarized by narration, frequency tables, histograms and pie charts. Multivariate analysis and Fisher exact tests were computed to determine the risk factors for associated injuries and their corresponding $95 \%$ confidence intervals were also calculated. A P-value less than 0.05 was considered statistically significant.

\section{Results}

Over the study period, a total of 1,092 paediatric patients were admitted to the orthopaedic ward of which $192(17.6 \%)$ were diagnosed with a femoral shaft fracture (Fig. 1). Of this group, 162 (84.4\%) femoral shaft fractures met the inclusion criteria to be in the final patient cohort for analysis, resulting in a femoral shaft fracture prevalence of $18 \%$.

Of this cohort, the median age was eight years and 72 patients (44.4\%) were aged between 6-12 years. Most fractures were seen in males $111(68.5 \%)$ with more than half of the patients being primary school children (Table 1). In addition, one-hundred forty-four (88.9\%) patients had no health insurance, and 64 patients $(39.5 \%)$ had fractures that occurred while along the road-side. 
Table 1

Patient Demographics $(\mathrm{n}=162)$.

\begin{tabular}{|c|c|c|}
\hline Characteristics & $\mathbf{N}$ & $\%$ \\
\hline Median Age in years (IQR) & $8(4-12)$ & \\
\hline \multicolumn{3}{|l|}{ Age category in years } \\
\hline$<1$ & 3 & 1.9 \\
\hline $1-2$ & 21 & 13.0 \\
\hline $3-5$ & 31 & 19.1 \\
\hline $6-12$ & 72 & 44.4 \\
\hline $13-18$ & 35 & 21.6 \\
\hline \multicolumn{3}{|l|}{ Sex } \\
\hline Male & 111 & 68.5 \\
\hline Female & 51 & 31.5 \\
\hline \multicolumn{3}{|l|}{ Level of Education } \\
\hline Preschool & 36 & 22.2 \\
\hline Kindergarten & 22 & 13.6 \\
\hline Primary & 86 & 53.1 \\
\hline Secondary & 18 & 11.1 \\
\hline \multicolumn{3}{|l|}{ Insurance status } \\
\hline Insured & 18 & 11.1 \\
\hline Not insured & 144 & 88.9 \\
\hline \multicolumn{3}{|l|}{ Location at time of injury } \\
\hline Home & 54 & 33.3 \\
\hline School & 13 & 8 \\
\hline Road & 64 & 39.5 \\
\hline Public playground & 18 & 11.1 \\
\hline Other & 13 & 8 \\
\hline
\end{tabular}

Most femur fractures were closed 156 (96.3\%). Eighty fractures (49.4\%) were mid-shaft femur fractures with a transverse fracture being the most common pattern. Skeletal traction was the most frequent inpatient fracture immobilization method observed. $69.1 \%$ of patients were treated Non-operatively with 104 (65.4\%) having longer than two weeks length of hospital stay (Table 2). 
Table 2

Patient Injury Characteristics ( $n=162$ ).

\begin{tabular}{|lll|}
\hline Characteristics & N & $\%$ \\
\hline Fracture type & & \\
\hline Closed & 156 & 96.3 \\
\hline Open & 6 & 3.7 \\
\hline Fracture location & & \\
\hline Proximal & 50 & 30.9 \\
\hline Middle & 80 & 49.4 \\
\hline Distal & 32 & 19.8 \\
\hline Side of femur fracture & & \\
\hline Right & 85 & 52.5 \\
\hline Left & 72 & 44.4 \\
\hline Both & 5 & 3.1 \\
\hline Fracture pattern & & \\
\hline Transverse & 79 & 48.8 \\
\hline Oblique & 34 & 21 \\
\hline Spiral & 106 & 65.4 \\
\hline Comminuted & 36 & 22.2 \\
\hline Type of traction & 33 & 20.4 \\
\hline Skin & 14 & 8.6 \\
\hline Skeletal & 53 & 32.7 \\
\hline Treatment Type & & \\
\hline Non Operative & 57.3 \\
\hline Operative & & \\
\hline Length Of Hospital Stay & & \\
\hline$\leq 7$ Days & & \\
\hline 8-14 Days & & \\
\hline 14 > & & \\
\hline
\end{tabular}


All children under one-year of age sustained a femoral shaft fracture from a fall at heights less than 2 meters. Toddlers (1-2 years) had most of their fractures result from falls of less than 2 meters followed by falling objects as the second most common mechanism of injury. Fractures resulting from RTIs were predominantly seen in older children, the 6-12 and 13-18 years of age categories, respectively. There was a significant number of children aged within 3-5 years age group whom sustained femoral shaft fractures as a pedestrian versus a motorcycle accident (MCA). Pedestrian RTIs was the leading mechanism of injury for this cohort of paediatric femoral shaft fractures (Table 3).

Table 3

Mechanism of Injury of Paediatric Femoral Shaft Fractures by Age $(n=162)$.

\begin{tabular}{|c|c|c|c|c|c|c|c|c|}
\hline \multicolumn{2}{|c|}{ Mechanism of Injury } & \multicolumn{5}{|c|}{ Age Categories in Years } & \multirow[t]{2}{*}{$\mathbf{N}$} & \multirow[t]{2}{*}{$\%$} \\
\hline & & $<1$ & $1-2$ & $3-5$ & $6-12$ & $13-18$ & & \\
\hline \multicolumn{9}{|l|}{ Fall } \\
\hline & Running & 0 & 6 & 5 & 4 & 3 & 18 & 11.1 \\
\hline \multirow[t]{3}{*}{$\leq 2 \mathrm{~m}$} & Playground & 0 & 2 & 2 & 8 & 8 & 20 & 12.3 \\
\hline & Furniture & 3 & 6 & 3 & 4 & 0 & 16 & 9.9 \\
\hline & Other & 0 & 1 & 5 & 7 & 3 & 16 & 9.9 \\
\hline$>2 m$ & Tree & 0 & 0 & 0 & 16 & 7 & 23 & 14.2 \\
\hline \multicolumn{9}{|l|}{ RTI } \\
\hline \multicolumn{2}{|c|}{ Pedestrian MCA } & 0 & 2 & 10 & 12 & 4 & 28 & 17.3 \\
\hline \multicolumn{2}{|c|}{ Pedestrian MVA } & 0 & 0 & 0 & 11 & 1 & 12 & 7.4 \\
\hline \multicolumn{2}{|c|}{ Pillion ${ }^{+}$MCA } & 0 & 0 & 3 & 3 & 4 & 10 & 6.2 \\
\hline \multicolumn{2}{|c|}{ Passenger MVA } & 0 & 0 & 1 & 2 & 5 & 8 & 4.9 \\
\hline \multicolumn{9}{|c|}{ Falling Objects } \\
\hline \multicolumn{2}{|c|}{ Gate/Doors/walls } & 0 & 1 & 1 & 2 & 0 & 4 & 2.5 \\
\hline \multicolumn{2}{|c|}{ Other objects } & 0 & 3 & 1 & 3 & 0 & 7 & 4.3 \\
\hline
\end{tabular}

$(+)$ - Pillion refers to a patient was carried on a motorcycle at the time of injury.

MCA - Motorcycle Accident.

MVA - Motor vehicle Accident.

A total of 38 (23.5\%) femoral shaft fracture patients presented with associated injuries, with traumatic brain injury (TBI) seen in 26 (69\%) patients in this group; and was the most common associated injury 
across all age groups. Most associated injuries were seen in the $6-12$ year-old group. Combined TBI, abdominal injury and femur fracture (Waddell's triad) was present in three (8\%) patients with associated injuries (Figure 2).

Children in the older age categories were more at risk of an associated injury compared to younger children. When comparing the age categories, the 6-12 year-old and the 13-18 year-old groups had 8 and 11 times higher odds for associated injuries, respectively. Females were $18 \%$ more likely to sustain associated injuries, although this was not statistically significant $(p=0.679)$. Patients with an open fracture had statistically significant more associated injuries $(p<0.001)$, while having a spiral fracture was protective against an associated injury (Table 4). Children with fractures because of a RTI exhibited 17 times higher odds of associated injuries compared to a fall. 
Table 4

Risk Factors for Femoral Shaft Fracture Associated Injuries $(n=162)$

\begin{tabular}{|c|c|c|c|c|}
\hline & \multicolumn{4}{|c|}{ Associated injuries } \\
\hline & Yes & Total & & \\
\hline & $\mathrm{n}(\%)$ & n (\%) & & \\
\hline Risk Factor & $38(23.5)$ & $162(100)$ & OR $(95 \% \mathrm{Cl})$ & $\mathrm{p}$-value \\
\hline \multicolumn{5}{|l|}{ Age (years) } \\
\hline$\leq 2$ & $1(2.6)$ & $24(14.8)$ & 1 & \\
\hline 3 to 5 & $7(18.4)$ & $31(19.1)$ & $6.71(0.76-58.87)$ & 0.086 \\
\hline 6 to 12 & $19(50.0)$ & $72(44.4)$ & $8.25(1.04-65.31)$ & 0.046 \\
\hline 13 to 18 & $11(28.9)$ & $35(21.6)$ & $10.54(1.26-88.31)$ & 0.031 \\
\hline \multicolumn{5}{|l|}{ Sex } \\
\hline Male & $25(65.8)$ & $111(68.5)$ & 1 & \\
\hline Female & $13(34.2)$ & $51(31.5)$ & $1.18(0.54-2.55)$ & 0.679 \\
\hline \multicolumn{5}{|l|}{ Fracture type* } \\
\hline Closed & 32 (84.2) & $156(96.3)$ & - & \\
\hline Open & $6(15.8)$ & $6(3.7)$ & - & $<0.001$ \\
\hline \multicolumn{5}{|l|}{ Fracture pattern } \\
\hline Transverse & $23(60.5)$ & $79(48.8)$ & 1 & \\
\hline Oblique & $8(21.1)$ & $36(22.2)$ & $0.69(0.27-1.75)$ & 0.441 \\
\hline Spiral & $2(5.3)$ & $33(20.4)$ & $0.16(0.03-0.71)$ & 0.016 \\
\hline Comminuted & $5(13.2)$ & $14(8.6)$ & $1.35(0.41-4.47)$ & 0.621 \\
\hline \multicolumn{5}{|c|}{ Injury Mechanism } \\
\hline Falling & $6(15.8)$ & $93(57.4)$ & 1 & \\
\hline RTI & $30(78.9)$ & $58(35.8)$ & $16.73(6.28-44.57)$ & $<0.001$ \\
\hline Falling objects & $2(5.3)$ & $11(6.8)$ & $2.64(0.47-14.71)$ & 0.269 \\
\hline
\end{tabular}

* Fisher's exact test

$\mathrm{Cl}$ - Confidence Interval

OR - Odds Ratio 


\section{Discussion}

\section{Prevalence}

The prevalence of femoral shaft fractures among paediatric orthopaedic admissions in this study was $18 \%$. This prevalence conforms to the reported Sub-Saharan prevalence observed in hospital-based studies conducted in Nigeria, Cameroon and Uganda which reported $10 \%, 16 \%$ and $22 \%$, respectively. ${ }^{13-}$ 15

Studies showing a lower prevalence of paediatric femoral shaft fractures in the literature, ${ }^{4,16,17}$ were population based retrospective studies in Europe and America and had a prevalence of $1.7 \%-5 \%$. The large difference in patient cohort size when comparing hospital-based and population-based studies could explain this discrepancy. Our hospital-based study might underestimate the true paediatric femoral shaft fracture prevalence for the region as some patients are missed due to treatment at other facilities or have sought treatment with a traditional bone setter.

We also found that males were more likely to sustain a femoral shaft fracture, as they represented 111 (68.5\%) patients in the cohort with a M:F ratio of 2.2:1. Our findings are similar to previous studies that showed male patients accounting for $62-73 \%$ of femur fracture patients. ${ }^{2,5,18}$ This result reflects the typical high risk behaviour exhibited by boys in Tanzanian society and requires an aggressive preventive strategy for this subset of the population.

\section{Mechanism of Injury}

In our study, the most common injury mechanism was a fall accounting for $57.4 \%$ (93) of all fractures and nearly half of these falls were in children in their home environment. Earlier studies also demonstrated falls as a common mechanism of injury. ${ }^{6,14,15,19}$ We found that most falls were from trees $(25 \%, 23)$, while other studies have documented that most occurred during playground activities. ${ }^{18,19}$ Regional variation in childhood recreational activities in addition to the habit of harvesting fruits and firewood from trees which is observed in our society might explain this difference.

Akinyoola et al. from Nigeria and Mughal et al. in South Africa reported RTI as the most common mechanism of injury throughout Sub-Saharan Africa. ${ }^{2,8}$ We found that RTIs resulting from a pedestrian versus MCA was the most common mechanism for sustaining a femoral shaft fracture accounting for $17.3 \%$ of overall childhood femoral shaft fractures. The absence of adequate infrastructure, poor traffic regulations and a lack of adult supervision most likely results in a higher risk of sustaining these injuries in the paediatric population. Child education on road safety and implementation of road safety rules is paramount to prevent a continued rise in the prevalence of fractures. 
Our data did not demonstrate the expected variation in the mechanism of injury across age groups. Other studies have shown a transition from falls to RTI as the major mechanism when shifting from younger to older children. Socio-economic activities in our region that involve tree climbing attribute the overrepresentation of falls from a height. ${ }^{18,19}$ In addition, 54 (33\%) of the femur fractures in our study occurred at home which poses a challenge as children are considered safe while at home due to theoretical adult supervision. This necessitates a need for public health interventions to further understand the risk, design appropriate family level education and prevent these injuries in the future.

\section{Associated injuries}

We found a $23.5 \%$ prevalence of femur fracture associated injuries that agree with other published studies, demonstrating a prevalence ranging from $28.6 \%$ in the USA to $36 \%$ in New Zealand. ${ }^{6,7}$ Being older than 6 years and being female increased the odds of sustaining an associated injury that is similar to findings reported in the literature. ${ }^{6,7}$ Older children are more likely to sustain a high energy injury mechanism, either a RTI or a fall from a height above 2 meters, which explains the predisposition to these associated injuries.

TBI was the most common associated injury in our patient cohort. Other studies have reported that other long-bone fractures are the most common associated injury followed by TBI. The use of helmets and protective equipment during contact or motorized sports which is mandated and paid attention to it in Western countries partly contribute to this. Sports related falls were the most common mechanism of sustaining a femur fracture in these other studies, while our study saw a higher incidence of RTI with pedestrian versus MCA $(17.3 \%) .{ }^{16,20}$

Fractures resulting from RTIs had an increased risk of having an associated injury that is similar to other studies in the literature. ${ }^{2,17} \mathrm{RTI}$ also increased the risk of Waddell's triad, which was $3(8 \%)$ of those with associated injuries in this study, similar to that seen in Nigeria (8.7\%) but significantly lower that seen in the USA (25.5\%). ${ }^{6,8}$ Understanding this association is critical in determining patient care requirements from the injury scene all the way until definitive hospital treatment as some associated injuries are life threatening if missed.

\section{Limitations and strength}

This study being retrospective could not control for the quality of the information found in the patient files and this resulted in 25 (13\%) of femoral shaft fractures being excluded from the study. The study established baseline data in paediatric femoral shaft fractures at our centre, which will help improve the management of these injuries.

\section{Recommendations}


Childhood femoral shaft fractures address a crucial component in children health and safety as these injuries often times are a result of high energy trauma as in this study pedestrian MCA and falling from trees were the top two leading specific mechanisms of injury, respectively. The need for a populationbased study exists to obtain a better understanding of this phenomenon, as other children continue to be treated at different facilities or traditional bone setters. More emphasis on road safety measures for drivers and pedestrians is critical as RTI attributed to most those with associated injuries and was the second leading cause of paediatric femoral shaft fractures.

\section{Conclusion}

Falls contributed to most femoral shaft fractures sustained in our cohort of paediatric patients, but pedestrian versus MCA was the leading mechanism of injury followed by falling from trees. The $18 \%$ femoral shaft fractures prevalence in our settings correlates with injury burden observed in other hospital-based studies within the region. TBI was the most common associated injury with RTI injuries contributing to the majority of them. It was established that being over the age of 6 years and being female increased the odds of having an associated injury.

The dataset(s) supporting the conclusions of this article is(are) included within the article (and its additional file(s).

\section{Declarations}

\section{Ethics approval and consent to participate}

The ethical clearance was obtained from the Kilimanjaro Christian Medical University College Ethical and Research committee with certificate number 2333. Confidentiality of patients was assured by using name initials and serial numbers. The committee waived the need for patient informed consent in this retrospective review.

\section{Declaration of Helsinki}

All procedures were performed in accordance with relevant guidelines' in the manuscript.

\section{Consent for publication}

All authors of the manuscript have read and agreed to its content and are accountable for all aspects of the accuracy and integrity of the manuscript in accordance with ICJME and authorize for its publication.

\section{Availability of data and materials}

All data generated or analysed during this study are included in this published article and is attached in supplementary information files. 


\section{Competing interests}

I declare that the authors have no competing interests as defined by $\mathrm{BMC}$, or other interests that might be perceived to influence the results and/or discussion reported in this paper. This manuscript is not under consideration for publication in any other journal.

\section{Funding}

The authors have not received any financial support for this work.

\section{Authors' contributions}

Conceptualization - Albert Macha, Rogers Temu, Honest massawe

Methodology - Albert Macha, Honest Massawe, Frank Olotu

Investigation - Albert Macha

Data curation - Frank Olotu, Albert Macha

Formal analysis - Frank Olotu, Albert Macha

Supervision - Honest Massawe, Neil Sheth, Rogers Temu

Writing Original draft - Albert Macha

Writing review \& editing - Albert Macha, Honest Massawe, Neil Sheth

\section{Acknowledgements}

The authors would like to thank the late Professor Augustine Mallya, the Department of Orthopaedics, Department of medical records and statistics from Kilimanjaro Christian Medical Centre for all their support during the development of this manuscript.

\section{Authors' information}

Corresponding author; P.o.Box 38645, Department of Orthopaedic and Trauma - Muhimbili Orthopaedic Institute, Dar es Salaam, Tanzania. Email: albertpaul2013@gmail.com.

\section{References}

1. WHO. WHO | Injuries and violence: the facts 2014. WHO. 2015.Accessed 2018 Jul 15;

2. Mughal MA, Dex-Peek SI HE. The epidemiology of femur shaft fractures in children Correspondence: SA Orthopaedic Journal Summer. 2013;12(4):23-7. 
3. Khoriati A-A, Jones C, Gelfer Y, Trompeter A. The management of paediatric diaphyseal femoral fractures: a modern approach. Strategies in trauma and limb reconstruction (Online). 2016;11(2):8797. Available from: https://doi:10.1007/s11751-016-0258-2

4. Mäyränpää MK, Mäkitie $O$, Kallio PE. Decreasing incidence and changing pattern of childhood fractures: A population-based study. Journal of bone and mineral research: the official journal of the American Society for Bone and Mineral Research. 2010;25(12):2752-9. Available from: https://doi:10.1002/jbmr.155

5. Petković L, Djan I, Gajdobranski D, Marić D, Petković M. [Pediatric femur fractures, epidemiology and treatment]. Vojnosanitetski pregled. 2011.Accessed 2018 Jul 29;68(1):9-14.

6. Rewers A. Childhood Femur Fractures, Associated Injuries, and Sociodemographic Risk Factors: A Population-Based Study. PEDIATRICS. 2005;115(5):e543-52. Available from: https://doi:10.1542/peds.2004-1064

7. Wilson NC, Stott NS. Paediatric femoral fractures: Factors influencing length of stay and readmission rate. Injury. 2007;38(8):931-6.

8. Akinyoola AL, Orekha OO, Taiwo FO, Odunsi AO. Outcome of non-operative management of femoral shaft fractures in children. African journal of paediatric surgery: AJPS. 2011 .Accessed $2018 \mathrm{Jul}$ 29;8(1):34-9. Available from: https://doi:10.4103/0189-6725.78666

9. Zaccai JH. How to assess epidemiological studies. Postgraduate medical journal. 2004.Accessed 2018 Jul 30;80(941):140-7. Available from: https://doi:10.1136/PGMJ.2003.012633

10. Jain A, Aggarwal A, Gulati D, Singh MP. Controversies in orthopaedic trauma-management of fractures of shaft of femur in children between 6 and 12 years of age. Kathmandu University medical journal (KUMJ). 2014;12(45):77-84. Available from: https://doi:10.3126/kumj.v12i1.13647

11. Jevsevar DS, Shea KG, Murray JN, Sevarino KS. AAOS Clinical Practice Guideline on the Treatment of Pediatric Diaphyseal Femur Fractures. Journal of the American Academy of Orthopaedic Surgeons. 2015;23(12):e101. Available from: https://doi:10.5435/JAAOS-D-15-00523

12. KCMC. Kilimanjaro Christian Medical Centre (KCMC) website. [Internet]. KCMC WEB PAGE. 2018.Accessed 2018 Jul 18.

13. Adewole O, Kayode M, Shoga M, Williams O, Ikem I. Pattern and Trauma Mechanisms of Paediatric Long Bone Fractures in Lagos, Nigeria. Nigerian Journal of Orthopaedics and Trauma. 2011.Accessed 2018 Jul 17;10(2):100-4.

14. Guifo ML, Tochie JN, Oumarou BN, Tapouh JRM, Bang AG, Ndoumbe A, et al. Paediatric fractures in a sub-saharan tertiary care center: a cohort analysis of demographic characteristics, clinical presentation, therapeutic patterns and outcomes. The Pan African medical journal. 2017;27:46. Available from: https://doi:10.11604/pamj.2017.27.46.11485

15. Hulme P. Mechanisms of trauma at a rural hospital in Uganda. Pan African Medical Journal. 2010;7:1-4.

16. Joeris A, Lutz N, Wicki B, Slongo T, AudigÃ L. An epidemiological evaluation of pediatric long bone fractures - a retrospective cohort study of 2716 patients from two Swiss tertiary pediatric hospitals. 
BMC Pediatrics. 2014;14(1):1-11.

17. Hoffmann CR, Traldi EF, Posser A. EPIDEMIOLOGICAL STUDY OF CHILDREN DIAPHYSEAL FEMORAL FRACTURES. Revista Brasileira de Ortopedia (English Edition). 2012;47(2):186-90. Available from: https://doi:10.1016/S2255-4971(15)30084-7

18. Loder RT, O'Donnell PW, Feinberg JR. Epidemiology and mechanisms of femur fractures in children. Journal of pediatric orthopedics. 2006.Accessed 2018 Jul 29;26(5):561-6. Available from: https://doi:10.1097/01.bpo.0000230335.19029.ab

19. Talbot C, Davis N, Majid I, Young M, Bouamra O, Lecky FE, et al. Fractures of the femoral shaft in children. The Bone \& Joint Journal. 2018. Accessed 2018 Jul 29;100-B(1):109-18. Available from: https://doi:10.1302/0301-620X.100B1.BJJ-2016-1315.R3

20. Tisherman RT, Hoellwarth JS, Mendelson SA. Systematic review of spica casting for the treatment of paediatric diaphyseal femur fractures. Journal of Children's Orthopaedics. 2018.Accessed $2018 \mathrm{Jul}$ 29;12(2):136-44. Available from: https://doi:10.1302/1863-2548.12.170201

\section{Figures}




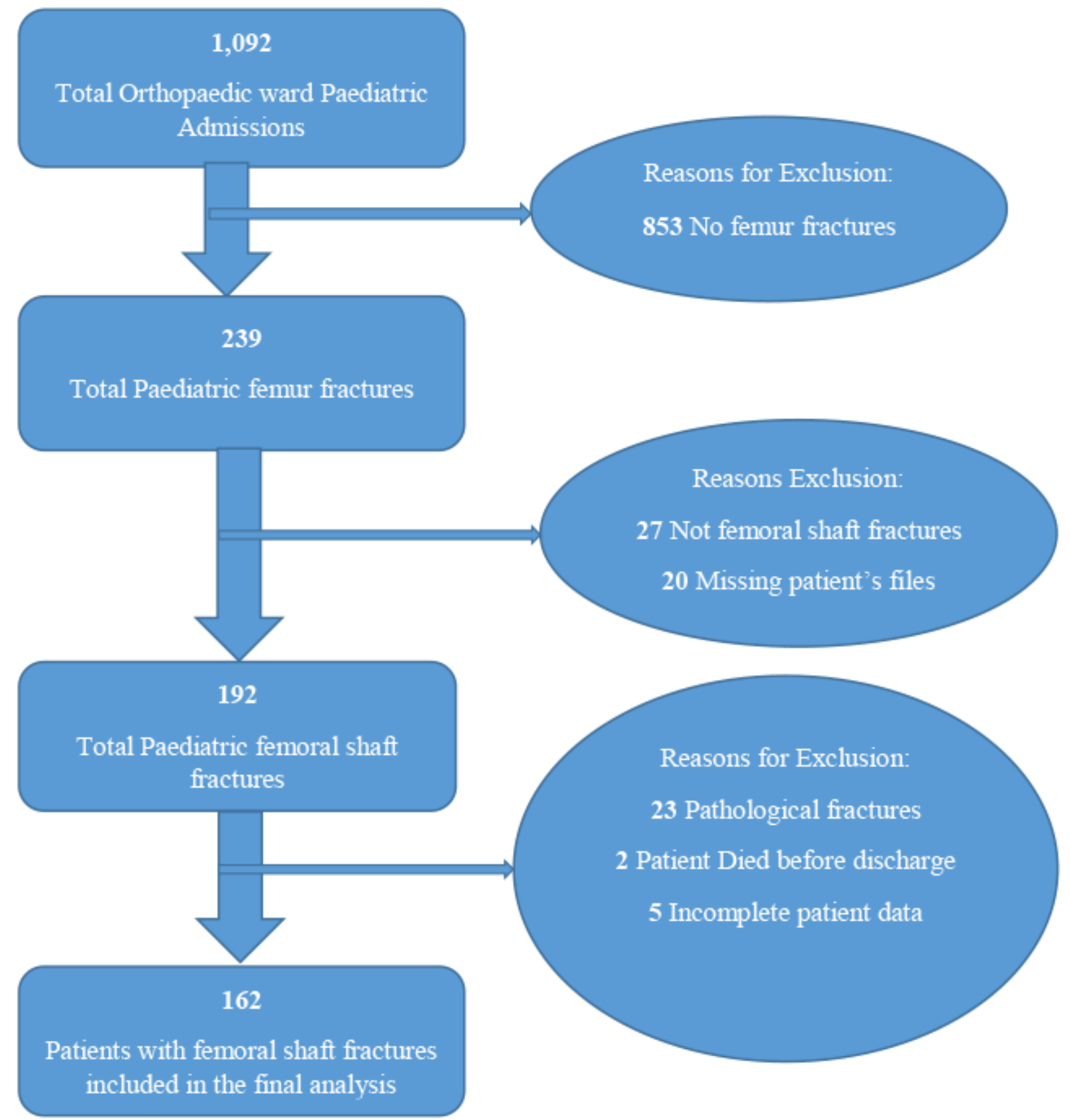

\section{Figure 1}

Femoral shaft fractures recruitment flowchart. 


\section{$\square$ TBI $\quad$ Other Fractures $\quad$ Soft tissue $\quad$ Chest $\quad$ Abdomen}

14

12

10

0
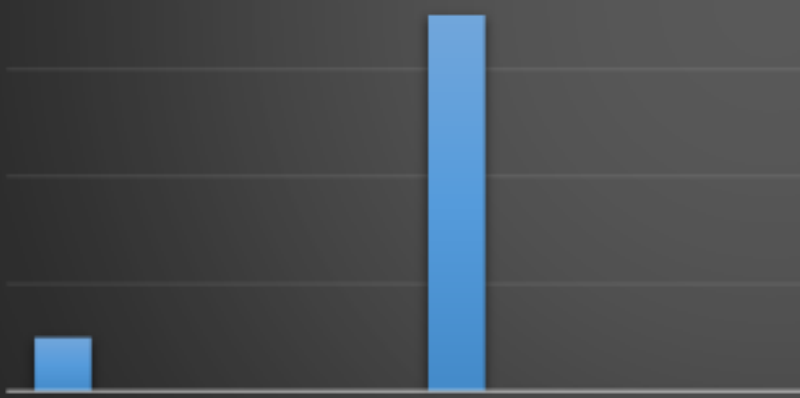

1 year - 2 years

3 years -5 years

6 years - 12 years

13 years -18 years

\section{Figure 2}

Distribution of associated injuries across different age categories $(n=38)$

\section{Supplementary Files}

This is a list of supplementary files associated with this preprint. Click to download.

- PaediatricFemurfracturesdataset.pdf 